\title{
Effects of boundary conditions on the critical spanning probability
}

\author{
Muktish Acharyya ${ }^{+}$and Dietrich Stauffer* \\ Institute for Theoretical Physics, University of Cologne \\ D-50923, Cologne, Germany
}

\begin{abstract}
:
The fractions of samples spanning a lattice at its percolation threshold are found by computer simulation of random site-percolation in two- and threedimensional hypercubic lattices using different boundary conditions. As a byproduct we find $p_{c}=0.311605(5)$ in the cubic lattice.
\end{abstract}

Keywords: Percolation, free boundaries, mixed boundaries, helical boundaries, threshold

+E-mail:muktish@thp.uni-koeln.de

*E-mail:stauffer@thp.uni-koeln.de 


\section{Introduction:}

In the case of random site percolation, the probability that the system is percolating below the percolation threshold $\left(p_{c}\right)$ is zero and it is unity above $p_{c}$. What is the probability of a cluster to span the lattice at the percolation threshold [1]? The earlier studies [2-6] tried to establish this quantity (spanning probability at percolation threshold) as an universal quantity. To check the robustness of the spanning probability, we have studied here the effect of different boundary conditions on the spanning probability at percolation threshold. Our study is confined to two and three dimensions and restricted to random site percolation.

For free boundary conditions, in three dimensions, the critical spanning probability is $0.265 \pm 0.005$ and it is universal [6]. However, an earlier study [3] gives different result $(\sim 0.42)$. This is due to the different boundary conditions used by two groups of researchers as pointed out in $[4,6]$. Here, we study the critical spanning probability (in two and three dimensions for random site percolation) and its dependence on the different boundary condition.

For clarity, let us first define our boundary conditions: In D-dimensions the spanning direction is always free

(1) Free boundary condition (FBC): The other D-1 directions are also free. (2) Helical boundary condition (HBC): The Helical boundary conditions are used in all D-1 remaining directions. (3) Mixed boundary condition (MBC): This is not applicable in 2 dimensions. In 3 dimensions, the Helical boundary condition is only applied in one of the directions of the planes and the other direction is free.

We are considering only site percolation. The microscopic connectivity is considered by the nearest neighbour occupied sites only and the macroscopic connectivity (spanning) is checked by standard Hoshen-Kopelman [1] algorithm. The spanning is checked only in one direction.

\section{Results:}

In $D=2$ we have taken $p_{c}=0.592746$ [1]. For a fixed system size $L$ we occupied the lattice randomly with probability $p_{c}$. We have used KirkpatrickStoll R250 random number generator as well as multiplication with 16807 [四]. 
Then we have checked whether the lattice is percolating or not by standard Hoshen-Kopelman algorithm [1] for $N_{s}$ number of different random samples. The number (fraction) of samples percolating at $p_{c}$ is called the critical spanning probability $\left(R_{p}\right)$. For a particular boundary condition, the spanning probability $R_{p}$ at $p_{c}$ has been calculated for different system sizes (for example, ranging from $L=17$ to $L=6551$ in two dimensions). We have calculated the critical spanning probability in 2 and 3 dimensions for the maximum allowable (in 128 MByte CRAY) system size. The spanning probability, $R_{p}$ (in two dimensions) has been plotted against $L$ for free boundary conditions (see Fig. 1). From the extrapolation, we found $R_{p}=0.501 \pm 0.003$. This is in good agreement with the earlier result $\left(R_{p}=1 / 2\right)[$ [ [ ] . The spanning probability for helical boundary condition in two dimensions was given earlier [9] as 0.637 and we report $R_{p} \sim 0.638$ in the asymptotic limit $(L \rightarrow \infty)$ (Fig.2).

In $D=3$, we have calculated the spanning probability $R_{p}$ at $p_{c}=0.3116$ [四]. Here, also we have calculated $R_{p}$ for different boundary conditions in the same way described above. Fig. 3 shows the plots of $R_{p}$ against $L$ for free boundary conditions. From extrapolation we obtained, $R_{p} \sim 0.28$, which is slightly higher than recently reported [6] value. Using the mixed boundary condition, we have calculated the critical spanning probability $R_{p} \sim 0.41$ in the asymptotic limit (Fig.4). This result is slightly smaller than the earlier estimate [3]. In the earlier case [3] the mixed boundary condition is used in the following way: the top layer is fully occupied, the helical boundary condition is used in one direction of the plane. But in the present case the top layer is randomly occupied with probability $p_{c}$, and the helical bounadry condition is used in one direction of the plane. These two slightly different boundary conditions give different result in the smaller systems, however in the $L \rightarrow \infty$ limit both boundary conditions give same result [7]. In addition, in three dimensions, we have calculated the critical spanning probability by using helical boundary condition in both directions of the planes. Our estimated value of $R_{p}$ using helical boundary condition is approximately 0.513 (Fig.5). In each case, in three dimensions, we observed that the value of $R_{p}$ is quite sensitive near $p_{c}$. For this reason we have shown some results by taking $p_{c}=0.31161$. All these results also indicate that $p_{c}$ lies in between 0.3116 and 0.31161 , compatible with earlier estimates [8].

In the present study, most of the data was obtained by taking $N_{s}=10000$ in each processor of a CRAY-T3E computer (using 64 processors). The CPU 
time required, for the whole study, can be calculated from the following example: for system size $L=481$ in three dimensions, each processor averaged over $N_{s}=800$ different random realisations. The total CPU time required for this is approximately 11 hours per processor.

\section{Summary:}

In summary, we studied the critical spanning probability in two- and three- dimensional system using different boundary conditions. Our results from numerical simulations (with larger system sizes and using better statistics) show that the critical spanning probability is strongly dependent on the boundary condition. There are different alternatives to helical boundary conditions in 3 dimensions, as pointed out in [6]. The value of the critical spanning probability is quite sensitive to the value of $p_{c}$ used. This has been shown in three dimensions and the size dependence of critical spanning probability can be an alternative tool for estimating $p_{c}$.

\section{Acknowledgements:}

Let us thank R. M. Ziff for important discussions, SFB 341 for financial support and HLRZ, Jülich for CRAY-T3E time.

\section{References:}

1. D. Stauffer and A. Aharony, Introduction to Percolation theory, Second edition (London: Taylor \& Francis) 1994

2. R. M. Ziff, Phys. Rev. Lett. 69 (1992) 2670

3. D. Stauffer, J. Adler and A. Aharony, J. Phys. A: Math Gen 27 (1994) L475

4. U. Gropengiesser and D. Stauffer, Physica A 210 (1994) 320

5. J. P. Hovi and A. Aharony, Phys. Rev. Lett. 76 (1996) 3874

6. C. Y. Lin, C. K. Hu and J. A. Chen, J. Phys. A: Math Gen 31 (1998) L111

7. R. M. Ziff, 1998, Private communication. 
8. C. D. Lorenz and R. M. Ziff, Phys. Rev. E, 57 (1998) 230; N. Jan and D. Stauffer, Int. J. Mod. Phys. C 9 (1998) 341; P. Grassberger, J. Phys. A: Math Gen 25 (1992) 5867; H. G. Ballesteros et al, Preprint (1998) cond - mat / 9805125

9. J. P. Hovi and A. Aharony, Phys. Rev. E 53 (1996) 235 


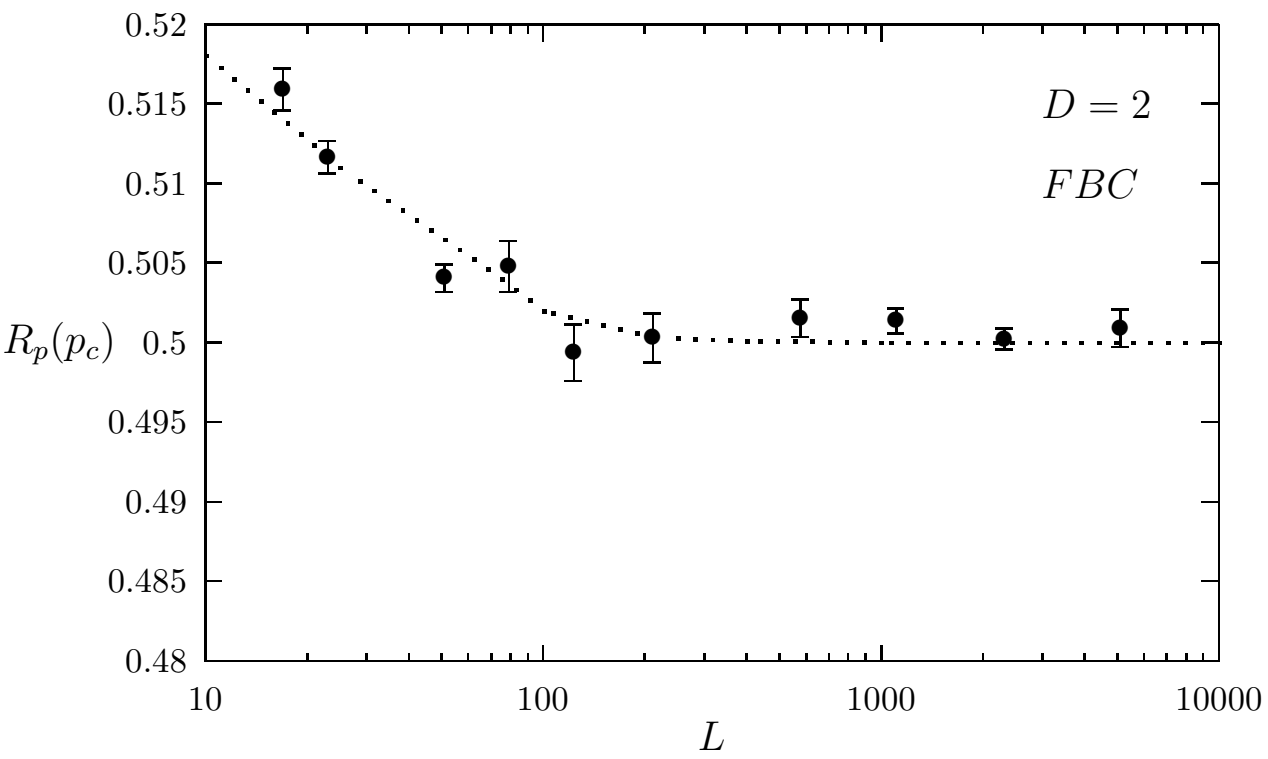

Fig. 1. Size dependence of critical spanning probability (using $p_{c}=0.592746$ ) in two dimensions for free boundary conditions. Dotted line is a guide to the eye.

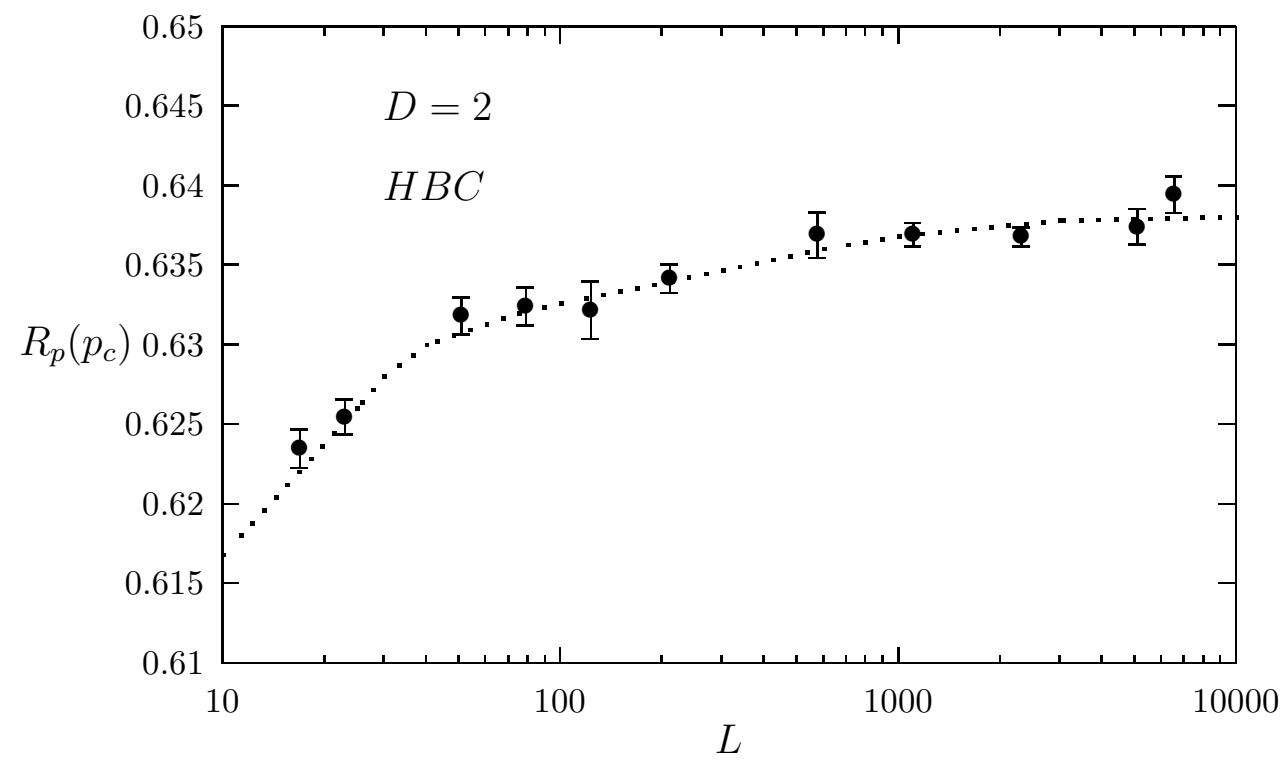

Fig. 2. As Fig. 1, for helical boundary conditions. 


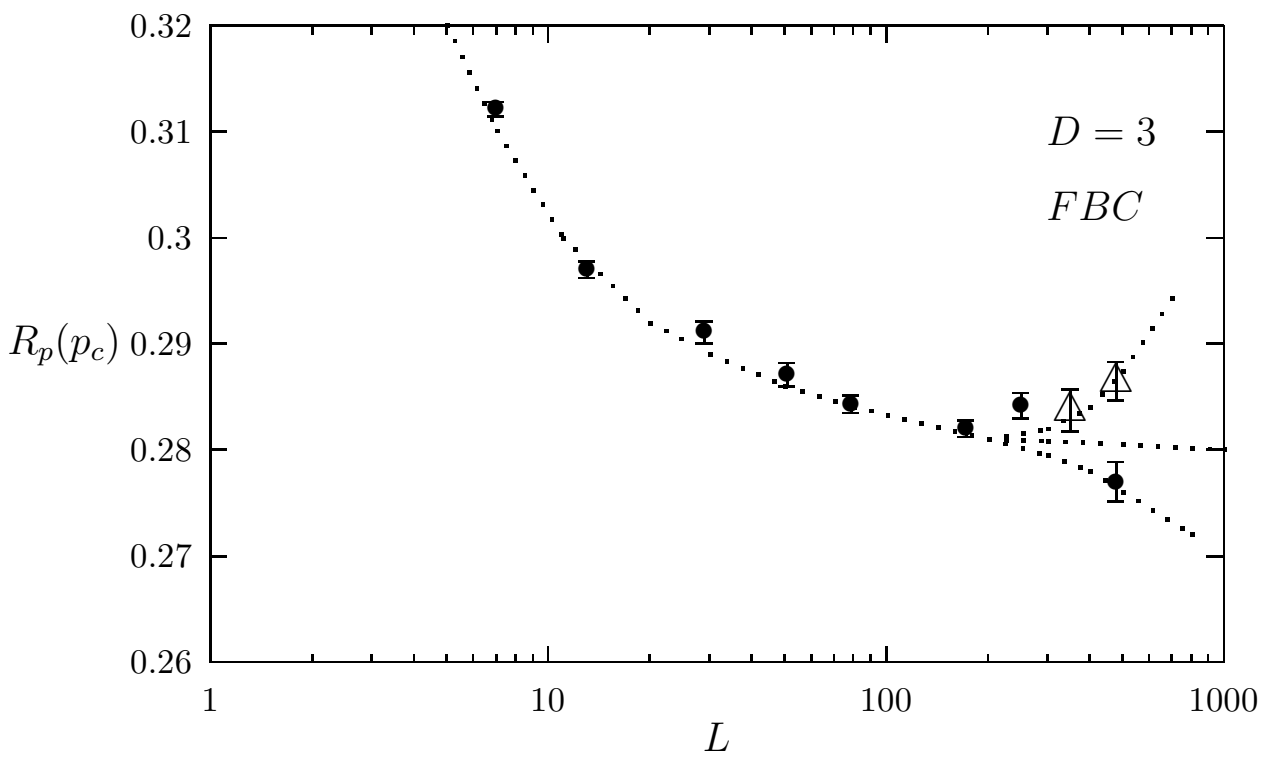

Fig. 3. Size dependence of critical spanning probability in three dimensions for free boundary conditions. The symbols $\bullet$ represent $p_{c}=0.3116$ and $\triangle$ represent $p_{c}=0.31161$. Dotted lines are guides to the eye.

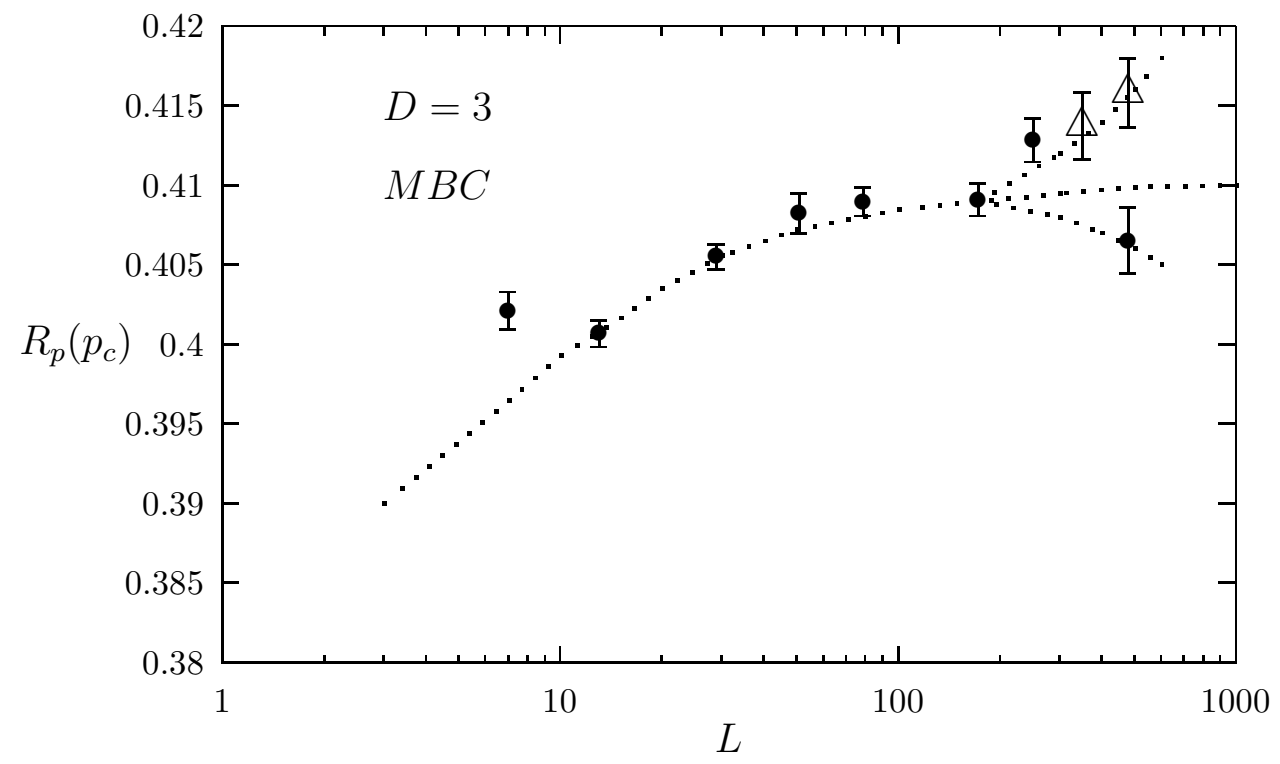

Fig. 4. As Fig. 3, for mixed boundary conditions. 


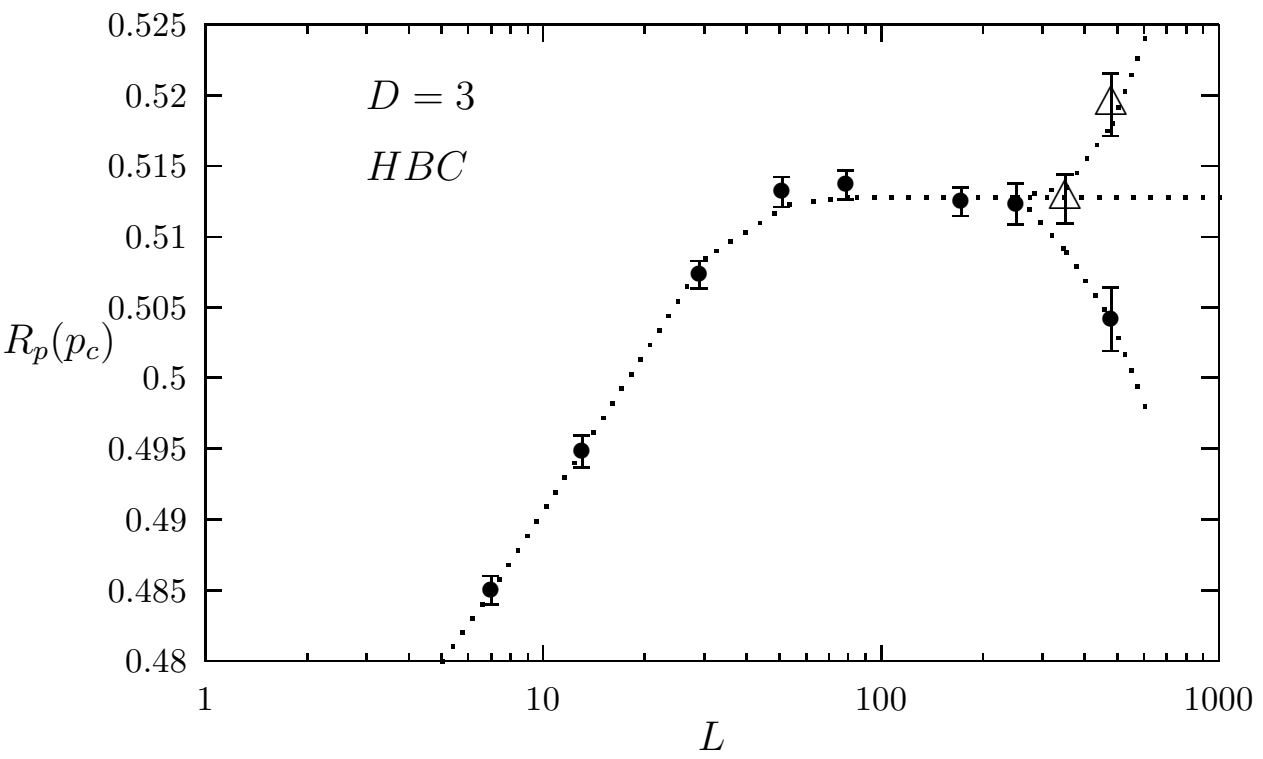

Fig. 5. As Fig. 3, for helical boundary conditions. 